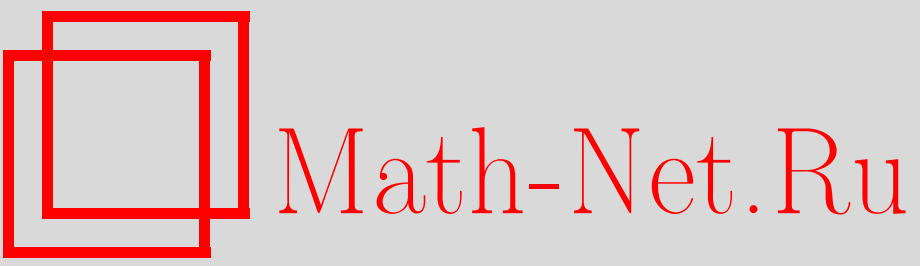

Ф. Ф. Воронов, Комплекс, порожденный вариационными производными. Лагранжев формализм бесконечного порядка и обобщение формулы Стокса, УМН, 1996, том 51, выпуск 6, 195-196

DOI: https://doi.org/10.4213/rm1024

Использование Общероссийского математического портала Math-Net.Ru подразумевает, что вы прочитали и согласны с пользовательским соглашением

http://www.mathnet.ru/rus/agreement

Параметры загрузки:

IP : 35.173 .137 .237

26 апреля 2023 г., 16:02:58 


\title{
КОМПЛЕКС, ПОРОЖДЕННЫЙ ВАРИАЦИОННЫМИ ПРОИЗВОДНЫМИ. ЛАГРАНЖКВ ФОРМАЛИЗМ БЕСКОНЕЧНОГО ПОРЯДКА И ОБОБЩЕНИЕ ФОРМУЛЫ СТОКСА
}

\author{
Ф. Ф. ВоРОнов
}

1. Основная конструкция. Пусть $M^{n \mid m}$ - гладкое (супер)многообразие. Рассмотрим лагранжианы $r \mid s$-мерных путей, т.е. отображений $\Gamma: I^{r \mid s} \rightarrow M^{n \mid m}$, где $I^{r \mid s}:=I^{r} \times \mathbb{R}^{0 \mid s}$, которые могут зависеть от производных произвольного порядка. Если лагранжиан зависит от производных порядка $\leqslant k$, то хорошо известно, что вариация действия, вообще говоря, содержит производные порядка $\leqslant 2 k$, и $\delta S=\int_{I^{r \mid s}} D t \delta x^{A}(t) \frac{\delta L}{\delta x^{A}}(x(t), \dot{x}(t), \ldots)$, где

$$
\frac{\delta L}{\delta x^{A}}:=\sum_{l=0}^{\infty}(-1)^{l}(-1)^{\widetilde{A}\left(\widetilde{F}_{1}+\widetilde{F}_{l}\right)} \frac{\partial^{l}}{\partial t^{F_{1}} \cdots \partial t^{F_{l}}} \frac{\partial L}{\partial x_{F_{1} \ldots F_{l}}^{A}} .
$$

(Отметим, что на лагранжианах произвольного порядка "полные производные" по временни́м переменным имеют смысл.) Для каждого лагранжиана $L$ определим новый лагранжиан $d L$, задающий действие для путей на единицу большей размерности:

$$
d L:=\dot{x}_{r+1}^{A} \frac{\delta L}{\delta x^{A}} .
$$

Теорема 1. Определение đ не зависит от выбора координат. Операция $d$ задает на пространстве всех лагранжианов структуру коцепного комплекса:

$$
\cdots \longrightarrow \Phi^{r \mid s} \stackrel{d}{\longrightarrow} \Phi^{r+1 \mid s} \stackrel{d}{\longrightarrow} \Phi^{r+2 \mid s} \stackrel{d}{\longrightarrow} \cdots .
$$

ДокАЗАтЕЛЬСтво. Независимость $\gtrsim$ от системы координат следует из того, что вариационные производные преобразуются как компоненты ковектора. Рассмотрим квадрат $d$. Непосредственное вычисление $d d L$ в общем виде затруднительно, так как требует явного раскрытия полных производных в формуле (1). Для лагранжианов первого порядка формальное выражение для $d^{2}$ содержит 36 слагаемых, которые действительно взаимно сокращаются, но проверка этого факта (автор ее проделал) мало приятна. Введем "взвешенные" пути и рассмотрим функционалы вида $S[\Gamma, g]=\int_{I^{r \mid s}} D t g(t) L(x(t), \dot{x}(t), \ldots)$, где $g$ - функция с компактным носителем в кубе $I^{r \mid s}$. Верна

Лемма. $\delta S=0 \partial \Omega g \forall g \forall \delta x(t): \operatorname{Supp} \delta x \cap \operatorname{Supp} d g=\varnothing \Longleftrightarrow \frac{\delta L}{\delta x^{A}}(x(t), \dot{x}(t), \ldots)=0$.

Рассмотрим действие $S^{*}$, отвечающее лагранжиану $đ L$. По определению вариационной производной

$$
\frac{\partial x^{A}}{\partial t^{r+1}} \frac{\delta L}{\delta x^{A}}(x(t), \ldots)=\frac{\partial}{\partial t^{r+1}}(L)+\text { производные по } t^{F}, \quad F \neq r+1 .
$$

Таким образом, множитель при $g^{*}\left(t^{*}\right)$ в интеграле есть "полная дивергенция", и $S^{*}$ переписывается в виде интеграла от $\pm\left(\partial g^{*} / \partial t^{F^{*}}\right) h^{F^{*}}\left(t^{*}\right)$ (аналог интеграла по границе). Для любой вариации $\delta x^{A}\left(t^{*}\right)$, удовлетворяющей условию $\operatorname{Supp} \delta x \cap d g^{*}=\varnothing$, получим $\delta S^{*}=0$, ибо носитель $\delta h^{F^{*}}$ не больше носителя $\delta x^{A}$. Применяя лемму, получаем $\delta \succsim L / \delta x^{A}=0$ тождественно, и $d d L=0$.

Когомологии комплекса (2) являются инвариантом гладкого многообразия $M$.

Работа выполнена при частичной финансовой поддержке Российского фонда фундаментальных исследований (грант № 96-01-01440). 
2. Обобщенная формула Стокса. Пусть дано семейство путей $\Gamma_{s}$ (постоянное вне компакта). Рассмотрим его как путь $\Gamma^{*}$, на единицу большей размерности.

Теорема 2. Для любого лагранжиана

$$
S\left[\Gamma_{1}\right]-S\left[\Gamma_{0}\right]=\int_{\Gamma^{*}} d L
$$

Если лагранжиан таков, что действие не зависит от параметризации (с нужным ограничением на ориентацию, см. [1]), то можно интегрировать по сингулярным многообразиям с границей и справедлив полный аналог формулы Стокса. Мы видим, что она не является исключительной принадлежностью дифференциальных форм. Формула (3) объясняет тождество $d^{2}=0$ как проявление общего факта "граница границы равна нулю".

3. Применение к теории форм на супермногообразии. Определение (1) впервые было дано автором в следующем контексте [2]: на супермногообразии (не являющемся чисто четным) было необходимо определить правильный аналог комплекса де Рама. В качестве форм было предложено рассматривать "ковариантные" лагранжианы первого порядка с дополнительным условием, обеспечивающим независимость вариации от ускорений (см. [1], [2]). В четном случае было показано, что воспроизводится теория Картана-де Рама. В общей ситуации теория нетривиальна. Указанное условие равносильно системе уравнений в частных производных [1, с. 58], нечетно-нечетная часть которых дает уравнения, известные при описании образа интегральных преобразований типа Радона [3], [4]. Введем фильтрацию лагранжианов по степени производных. Тогда наши уравнения получают "гомологическую" интерпретацию как условие сохранения фильтрации под действием дифференциала $\Varangle$ и очевидные высшие аналоги. Результаты настоящей заметки дают существенное упрощение доказательтв [1]. Недавно построена "вторая половина" комплекса форм на супермногообразии на дуальном языке [5]. Можно надеяться, что сочетание методов [5] и настоящей работы приведет к новому продвижению.

4. Применение к обратной задаче вариационного исчисления. В соответствии с теоремой 1 , чтобы проверить, что набор функций $f_{A}$ является вариационными производными от лагранжиана $r \mid s$-путей, необходимо составить $d\left(\dot{x}_{r+1}^{A} f_{A}\right)$ и проверить равенство нулю. (Заметим, что сечения расслоений также могут быть интерпретированы как "поверхности" или "пути".) Обратная задача вариационного исчисления изучалась в [6], [7] и др. Было бы интересно сопоставить подход этих работ с комплексом (2).

В заключение можно заметить, что известные в теории гомологий конструкции дифференциалов сводятся к двум или трем основным классам (комбинаторный и дифференциалы типа Картана или Кошуля). "Вариационные" дифференциалы, изучаемые в [1], [2], [5] и настоящей работе, по-видимому, дают еще один, новый, класс.

\section{СПИСОК ЛИТЕРАТУРЫ}

[1] Voronov Th. Geometric integration theory on supermanifolds // Soviet Sci. Rev. Sect. C. Math. Phys. Rev. 1992. V. 9. [2] Воронов Ф.Ф., Зорич А. В. // Функц. анализ и прилож. 1986. Т. 20. № 2. С. 58-59. [3] John F. // Duke Math. J. 1938. V. 4. P. 300-322. [4] Гельфанд И. М., Гиндикин С. Г., Граев М. И. // Итоги науки и техники. Соврем. пробл. матем. Т. 16. М.: ВИНИТИ, 1980. С. 53-226. [5] Voronov Th. dg-ga/9603009. Topics in Geometry and Mathematical Physics // AMS Translations / ed. S.P. Novikov, 1996. [6] Vinogradov A. M. // J. Math. Anal. Appl. 1984. V. 100. №1. P. 1-129. [7] Tulcziew W. // Bull. Soc. Math. France. 1977. V. 105. P. 419-431.

Московский государственный

Принято редколлегией университет им. М. В. Ломоносова 03.10.1996

E-mail: theodore@mech.math.msu.su 